\title{
HOLONIC MANUFACTURING WITH INTELLIGENT OBJECTS
}

\author{
Eddy Bajic, Frédéric Chaxel \\ Research Center for Automatic Control, University of Nancy \\ CRAN - CNRS UMR 7039 \\ BP 239, 54506 Vandoeuvre-les-Nancy, FRANCE \\ eddy.bajic@cran.uhp-nancy.fr \\ frederic.chaxel@cran.uhp-nancy.fr
}

\begin{abstract}
This paper presents and analyzes different approaches in the implementation of the Intelligent Manufacturing System concept, with a main focus on the holon paradigm proposed by the HMS project. After a survey of manufacturing systems evolution, it deals with the holon-product concept and its instantiation to the physical part product as a key issue to novel manufacturing system. Propositions are discussed to enrich products with new capabilities in order to decide and deal actively with their environment, such as intelligent objects. Several methodologies are described to implement this approach and an original one is proposed to promote holonic control of production systems driven by intelligent products. New perspectives of process reactivity and robustness, product tracking, and product life cycle information management are depicted, as also problems still not solved.
\end{abstract}

\section{INTRODUCTION}

Since the last decade, tremendous changes have occurred in the field of manufacturing systems research activities considering production control and organization. To manage product diversity and rapid changes in production demands, research effort of the nineties have been focused on manufacturing systems architectures satisfying productivity, flexibility, openness characteristics for integration aspect and cost reduction, widely based on automation increase (Dilts, 1991).

Originally represented in the eighties by the CIM concept (Computer Integrated Manufacturing), new approaches emerged to remedy to the limits hit by such hierarchically and centrally controlled organization suffering from rigidity in response to fast production changes and adaptations. Then, priority have been put on decentralization and distribution of decision-making activities and information flow with an expected downsizing of manufacturing systems. Fully distributed structures were proposed (Duffie, 1986) involving concurrent mechanism for job decision and coordination support, based on network communication procedures, contract nets. Although heterarchical architectures were promoted to provide manufacturing systems with decentralization, modularity, robustness and cooperative functionnalities. 
Beyond those approaches characterized by system-based functionality, and machine-centered decision making, strong demand appears for new formalizations of manufacturing systems to break off from conventional system organization to satisfy autonomy and individualism along with cooperation capabilities amongst all components of a manufacturing system. Emphasis was put on coordination, selforganization, hyper-flexibility, adaptation and product-orientation.

\section{NEXT GENERATION MANUFACTURING SYSTEMS}

Several new paradigms for manufacturing system description were formulated drawing inspiration from the real world mainly over the two aspects of social organization of human societies and natural rules and environment.

Biological metaphor have been proposed by Okino (Okino, 1994) to fit autonomous distributed manufacturing system description, so called Bionic Manufacturing System (BMS) in CAM-L/Japan. BMS concept lays on a system theory involving self-organization rules over distributed components with quasi-life functions copying genetic behavior, allowing freely adaptive and flexible connections in response to changes of system conditions. Ueda (Ueda, 1994) specified a model of BMS as a pseudo ecosystem built around works and manufacturing cells thus mimicking biological organisms carrying DNA (Deshoxyribo Nucleic Acid) gene type and BN (Brain and Neuron) type behavior and information. In a Bionic world environment, works and tasks are considered as organisms that grow up to become or generate products, manufacturing cells are other types of organisms that process works. DNA type information is supporting inherited information and transformation as "growing up" objectives of products, while BN type information governs works activities. In this, a manufacturing system control behavior should be described by evolution strategies with mathematical model based expressions.

A social organization metaphor has been derived from the socio-philosophical proposition of a Hungarian journalist and philosopher Arthur Koestler, specified early in 1971 in the book "The ghost in the machine", as the holonic concept (Koestler, 1989). Based on the observation of self-regulation capabilities of social organization, a social holonic behavior involves very few characteristics supported by elementary entities named Holon, acting and cooperating to assume living of complex and ruled organization named Holarchy. Koestler adds that holonic argument can be applied to biological, social or cognitive hierarchy which manifests rule-governed behavior and structural consistency.

The holonic metaphor have been applied to manufacturing system organization by the International joint program IMS (Intelligent Manufacturing System) with the thematic research consortium HMS (Holonic Manufacturing System) as a promising response to provide production systems with robustness and adaptability to condition changes and disturbances, modularity and flexibility capacities. Underlying principles of holonic application to manufacturing systems have been defined as follows by the HMS consortium (Van Brussel, 1995) : Holon : An autonomous and co-operative building block of a manufacturing system for transforming, transporting, storing information and physical object. Holarchy : An assembly of holons which act in cooperation having a specific set of objectives and 
common goal. Autonomy : Ability of an holon to control its own execution plan associated with its own strategy. Co-operation : Capabilities of systems entities to communicate, negotiate and execute actions plans in order to reach a global objective.

The previous paradigms are actually drawing up the next generation manufacturing systems to come. Scientist research community have to cope with formalization and resolution of the aiming capabilities of the real world to validate and prove efficiency of metaphorical paradigms and concepts within the production world. Agent technology derived from artificial intelligence research fields have emerged to support distributed intelligent control within manufacturing system architecture (Jennings 1995, Shen 2000). So the Holon, previously an imitation metaphor of social and human organization has become consequently a pragmatic element of agent technology implementation represented by an intelligent software agent (Fletcher, 2001).

A major goal is to fit the abstract world mainly focused on information management, treatment and decision-making capacities, to the real world represented by physical product-parts, machines and resources. By joining bits to atoms, BMS and HMS paradigms could take shape. In that way the need for distribution of information system onto individual product and objects requires tools and methods to manage and provide automatic or human-based interaction to product related information for manufacturing and logistic systems.

This conducts to a conceptual dilemma to answer : does the product itself need to be intelligent, or instead do intelligent services have to be offered to the product ? The following paragraphs analyze different solutions and focus on a validation approach of the holon concept supported by part product and resources cooperating in a manufacturing environment as intelligent objects.

\section{PRODUCT ORIENTED MANAGEMENT}

A common characteristic is revealed in both approaches BMS and HMS, with the duality of elementary organisms performing in complex systems. A holon consists of an information processing entity allied to a physical processing entity, as also biological organisms are unified entity of information and substance. This characteristic contributes to the emergence of an autonomous behavior appearance in complex systems, on the basis of internal structure of holon as described in Figure 1. The holon-product as an intelligent object concept can be seen as an entity created by real or forecasted market demand, ruling a servicing environment in a competitive and cooperative manner. At the first stage, the intelligent object should embed the necessary knowledge to assure the realization of the associated product, with the capacity of disseminating individual customer and product specifications directly within the process.

An essential characteristic of the described holon-product formalization is the merging of bits and atoms on one host, the product, in order to maintain consistency and coherency in between abstract world (manufacturing plan order and scheduling ) and real world (material flow, physical production process or distribution environment) (Brock 2001).

Product information and knowledge is basically defined at the design level in 
words of product specification and process operations as quality criteria, manufacturing plan, maintenance procedure, ... This knowledge need to be constructed step by step and structured through a life cycle product information model, to be shared by all the actors tools operating on the product. At the manufacturing stage, this knowledge is conceptually duplicated to fit to each individual product, so that product definition data set is cloned, and then follow its own life.

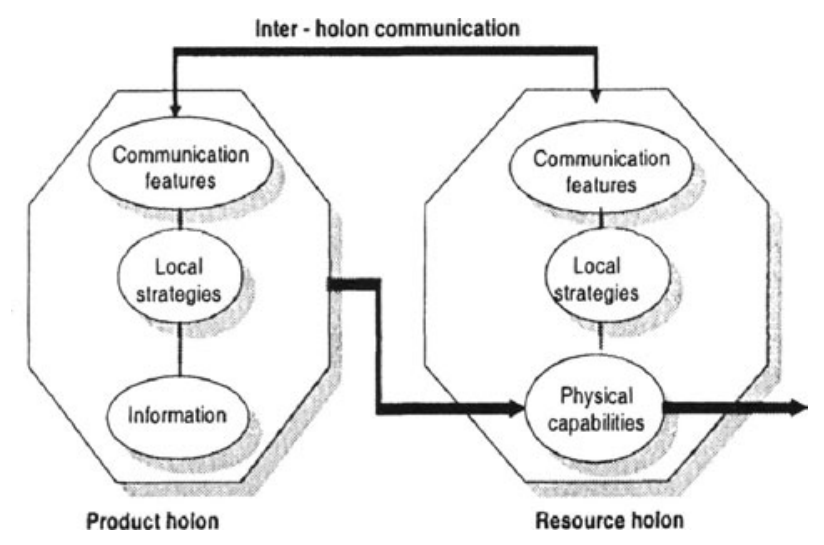

Figure 1 - Resource and Product Holons structure

In the intelligent object approach, a product as a holonic entity object carry its own information on a programmable electronic tag (R/W mode) communicating with each user involved in its development process (Bajic 1999, MacFarlane 2000). Advantage of this approach is in machine capacities to focus on operations control (transport, measures, ...) and not on coherency maintenance of product related information, this point is conceptually assumed by each individual product. A dialog can be imagined in between machine and product as follows : "Part 17843, where are you ?" says the machine, "I am currently at Machine 4 for a 10 minutes operation and I expect to be soon served by you" replies the product. This leads to the emergence of claims contracts between customers and suppliers in a concurrent client/server formalism.

Thus intelligent Tracking in manufacturing and supply chain environment is affordable, offering two new concepts for product mobility management known as postponement and merge-in-transit (Brewer 1999). Postponement is the capability to assign destination or final realization of a general purpose object (i.e. a semifinished product) at the latest time in the process when production availability or client demand occurs. Merge-in-transit involves combining various objects (productparts to be assembled), thus merging information system and objects capabilities at a common place to be delivered as a whole to the customer site.

The major issues of the proposed intelligent object approach lie on one hand in the management of information and object mobility, and on a second hand on decision-making capabilities associated to each holon-product. Automatic identification technologies using electronic data carriers and radio frequency techniques have now gain in maturity and have been industrially proofed, so they are currently the target technologies to implement attended functionality to the product. But still limitations in these technologies, mainly du to the current market size, 
bounds the physical implementation of the holon-product concept, by mean of lack of storage capacity (up to Mbyte needed by automotive application Bajic 1999), and lack of user-program and method encapsulation within tag. Nevertheless the today technological barrier, enhancement and refinement of the intelligent object concept still have to be continued with the objective of a full embedding on the product.

As results of research conducted at CRAN since several years, an intelligent product design and formalization methodology, still under development is proposed in the following chapter as a logistic support to manufacturing system control. The following chapters shows part of this methodology concerning holon-product data management. Holon-Product specification will assume both vertical integration up to the design level and the horizontal integration over the process organization by supplying methodology and support tool allowing each part carrying electronic tag, to act as a communication vector of process information system, in charge of the overall application management, and information coherency, consistency and accessibility.

\section{PRODUCT DRIVEN PROCESSING}

In the way to reach the formalization of the holon-product concept as an intelligent object, individual an dmobile information management is one of the key challenges. For this purpose, international research activities currently running are investigating two approaches.

The Auto-ID center (Brock 2001) based at MIT Lab and Cambridge University UK, propose an information system infrastructure centered on the product-part which is tagged with a 96 bits RFID smart tag. Products are virtually connected to internet, where life cycle product information is stored and indexed by mean of a code number named ePC (electronic product code) tagged on the product, and representing a pointer to distributed data bases over internet network (Figure 2). The ePC approach works together with a Product Markup Language (PML), describing the object with XML (eXtended Markup Language) syntax and Object Naming Services (ONS) implemented on Internet machines, thus assuming the routing of product information request to appropriate distributed web sites as Domain Name Servers actually do in Internet philosophy. The Auto-ID project is mainly focused on retail supply chain applications with low cost passive tag.

Research on intelligent product concept conducted at CRAN tends to implement information management capacity directly on the product, assuming Mobile Database Nodes infrastructure for intelligent objects management. Figure 3 depicts the concept adapted from a client/server local model, where the object is the manager of its own information and gives them to the process after it received a service request ("What is the next operation to be proceed on yourself" for example).

Requests issued from the process are sent to an object interface application supporting product access methods in an object-oriented requesting semantic such as Object_Selector.Message (Parameters), SystemMessage(Parameters): color.getvalue(); color.putvalue(Red); 


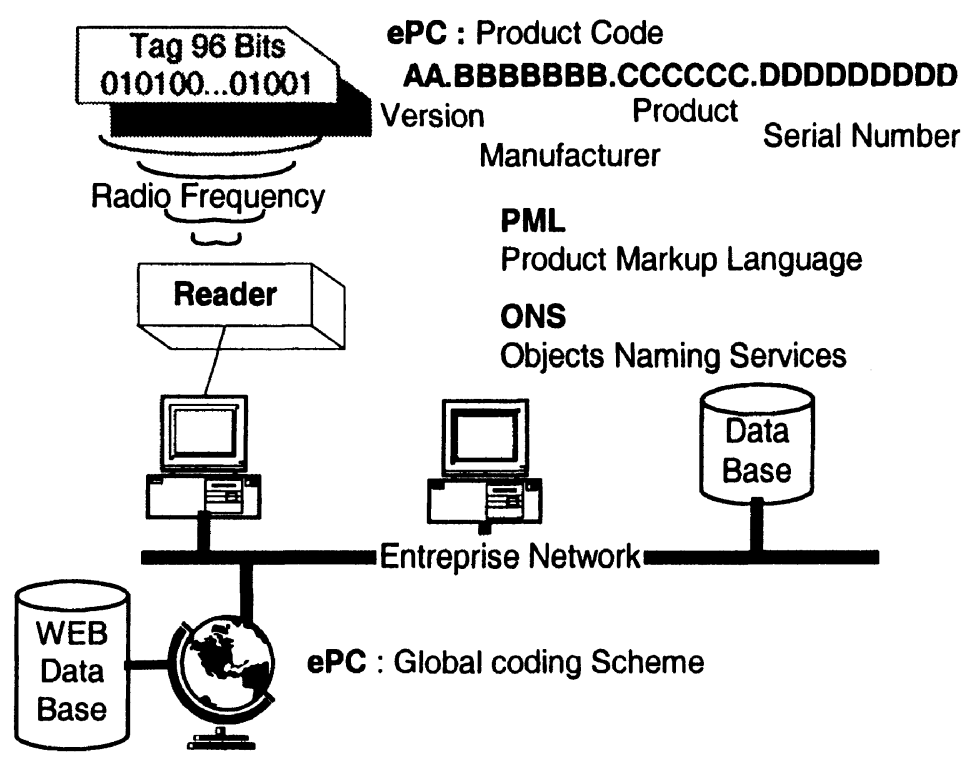

Figure 2 - ePC product-tagged information infrastructure

A the STEP-based methodology (Standard for Exchange of Product data Model) allows translation of product models, described in the EXPRESS language into :

- a library of product-information access functions, allowing easy applicationoriented requesting of product according to the EXPRESS data model;

- an external repository database structure definition, to assume a full description of product information model when storage capacity on the product is limited. This is an optional service of the methodology.

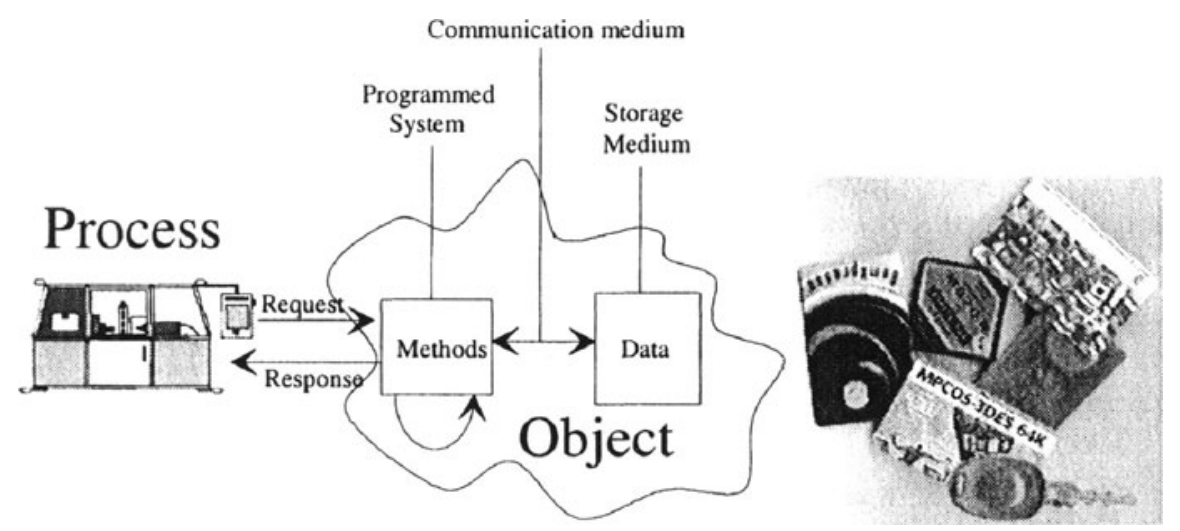

Figure 3 - Intelligent object in manufacturing system \& RFID tags embedded

The product definition within the STEP neutral file is used on one hand to create the tag memory image, for the first state of a product associated to the current phase of its life cycle in the manufacturing environment, and on a second hand if needed, to populate the previously created database schema. Although holon-product definition is the result of a first phase supplying both a holon-product data model and holon data in the form of an EXPRESS data model and STEP files. Product 
information is split into two categories, one is managed on the product and the other is distributed over a networked database as shown on Figure 4.

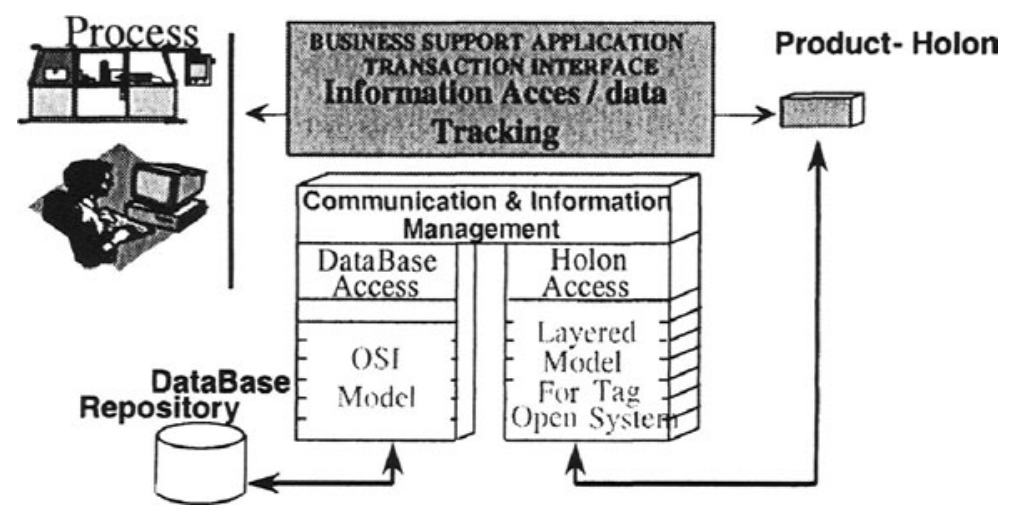

Figure 4 - Dual access to intelligent product data models

This information dichotomy of the proposed methodology firstly assumes a high degree of autonomy to assume product-based process management, with timeresponse efficiency for real time process activity on the object independently of any information computing complex infrastructure, and secondly it allows accessibility to large volume of data to cover product life cycle management, according to product data models stored on the product. Figure 5 shows the implementation process assuring intelligent object management and information system distribution over both the product and distributed database.

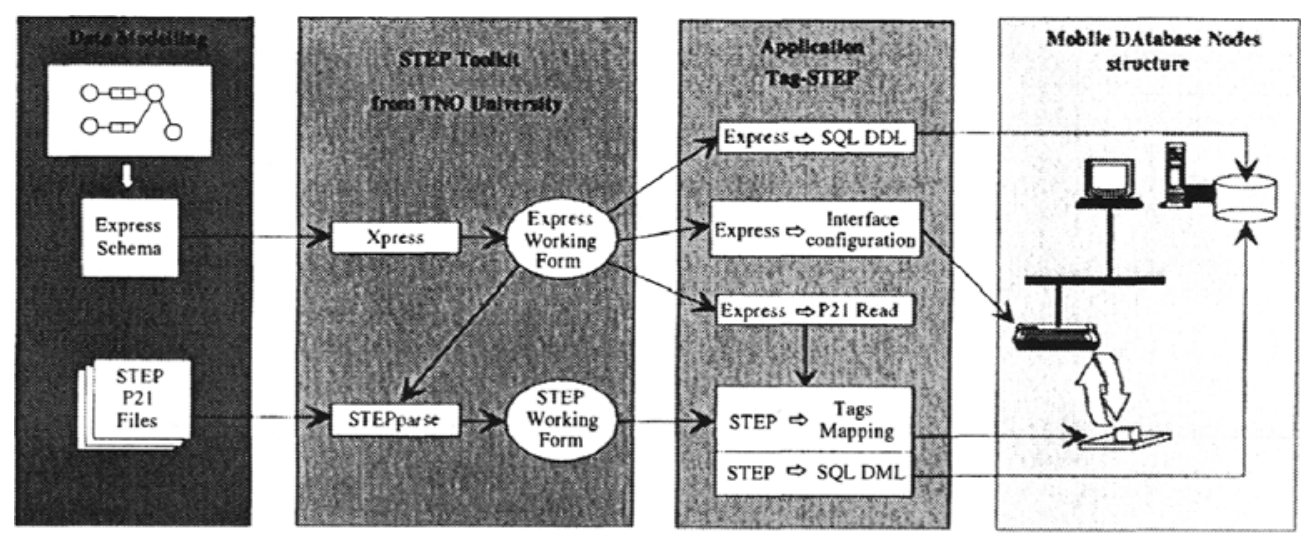

Figure 5 - Product Information system implementation processing

Current storage capacity of modern tags offers up to 128 Kbytes, which can be considered as huge but often not enough as regard to the needs in automotive industries for example, and at expensive price per part. The dichotomic methodology presented before have been successfully validated in automotive application with Renault car manufacturer research project resulting in no-key car vehicle Laguna II (Bajic 2002). 


\section{CONCLUSION}

The intelligent object concept is promising significant benefits in automation applications to cover the full product life cycle management from production up to after sale services and recycling. Manufacturing system control activities and supply chain management are the meaningful targets. From the concept to its instantiation, several approaches are currently investigated providing different ways for distribution of competencies in between the product and its environment. Manufacturing automation and devices are certainly not yet matured enough to integrate such innovative control and give intelligence to the product. Cost matter is currently orienting research activities towards less intelligence on product, but robustness, reactivity, and object autonomy suffer from this. But there is still limitations in the Auto-ID technologies, mainly due to a small market size, bounds the practical implementation of the intelligent object concept performing holon product metaphor, that needs incorporation of computing power into tags. Applications have been investigated by the authors with smart java card, with virtual java machine inside the object, promising high efficiency. Conceptual development still have to be enhanced, with a need for formal modelling of intelligent product, independently of technological platform. Even multi-agents technology do not give yet formal solution, without deep embedding in software platform.

\section{REFERENCES}

1. Bajic E, Chaxel F, Auto-ID Mobile Information System for Vehicle Life Cycle Data Management, IEEE SMC, October 6-9 2002, Hamamet, Tunisia

2. Brewer A, Sloan N, Intelligent tracking in manufacturing. Journal of intelligent manufacturing, 1999, 10: pp 2 45-250.

3. Brock D, The electronic product code. Auto-Id center, MIT, Cambridge, 2001.

4. Bajic E, Chaxel F, Richard J. Automotive vehicle data management based on holon-product paradigm IEEE SMC, pp 122-132, October 12-15 1999, Tokyo, Japan.

5. Dilts D.M., Boyd N.P., (1991). Whorms H.H. The evolution of control Architecture for Automated Manufacturing systems. In Manufacturing Systems, Vol 10, $\mathrm{N}^{\circ}$ 1, pp 79-93.

6. Duffie N. A. , Piper R. S. . Non-hierarchical Control of Manufacturing Systems. In Manufacturing Systems, 1986, Vol 5, No 2, pp 137-139.

7. Fletcher $M$, Deen $M$. Fault tolerant holonic manufacturing systems. Concurrency and computation : practice and experience, 2001, 13:43-70.

8. Jennings $\mathrm{N}$. Coordination techniques for distributed artificial intelligence. Foundations of distributed intelligencec ch. 7, O'Hare GH, Jennings N (Eds); Wiley, 1995

9. Koestler A. The ghost in the machine, Arkana books, 1989, London.

10. Lonc B., Bajic E. "Design and exploitation of communicating escort memories for automotive applications". Int. conf. on Advanced Microsystems for automotive applications, VDI-VDE-IT, pp. 142-150, 19 96, Berlin, Germany.

11. MacFarlane D, Bussmann S. State of the art of holonic systems in production planning and control. Production planning and control, 2000, V11, N6,: 522-536

12. Okino, N. Bionic manufacturing system. In Manufacturing Systems, 1994, Vol. 23, pp. 175-187

13. Shen W, Maturana F, Norrie D. Metamorph II : an agent based architecture for distributed design and manufacturing. Journal of Intelligent manufacturing 2000: 11, 237-251.

14. Ueda K . Biological-oriented paragidgm for artifactual systems. Japan symposium on flexible automation, Kobbe, 1994, pp 1263-1266

15. Van Brussel H., Wyns J., Valckenaers P., Bongaerts L., Peeters. Reference architecture for holonic manufacturing systems : PROSA Computers in industry, 37, N³, pp225-276, 1998 